\title{
Legitimationsstrategien von Autokratien
}

\section{Eine Einführung}

\author{
Steffen Kailitz $\cdot$ Stefan Wurster
}

Online publiziert: 18. April 2017

(C) Springer Fachmedien Wiesbaden 2017

\section{Legitimation von Macht}

Die jüngste Welle der Autokratieforschung hat viele neue Erkenntnisse über Autokratien zu Tage gefördert (vgl. statt vieler Geddes 1999; Gandhi 2008; Svolik 2012). Sie nahm allerdings doch etwas einseitig Institutionen und ihre Kooptation in Autokratien in den Blick (Schedler 2009; Pepinsky 2013), der Aspekt der Legitimation autokratischer Herrschaft wurde zunächst weitgehend ignoriert (Kailitz und Köllner 2013; Köllner und Kailitz 2013). Angesichts der zentralen Bedeutung des Legitimationsaspekts in zahlreichen Strömungen der Politikwissenschaft ist es nicht verwunderlich, dass nach und nach immer mehr Stimmen in der Autokratieforschung forderten, den Aspekt der Legitimation deutlich stärker zu berücksichtigen (Backes 2013; Burnell 2006; Gerschewski 2013; Holbig 2013; Kailitz 2013a; Kneuer 2013; Pickel 2013; Schlumberger 2010; Kailitz und Stockemer 2015; Abulof 2015; Morgenbesser 2015; Hanson 2011; Brusis 2016; Dukalskis 2017). Mit Blick auf die Vergleichende Politikwissenschaft handelt es sich bei der Frage nach den Legitimationsstrategien von politischen Regimen (vgl. statt vieler Connolly 1984; Denitsch 1979; 1976) schließlich um ein Kernthema. Für Max Weber (1980, 1984), einen der wichtigsten Wegbereiter der modernen Sozialwissenschaften, war die Legitimation politischer Herrschaft gar das Schlüsselkriterium zum Verständnis politischer Herrschaft. Auch aus der von Max Weber inspirierten systemtheoretischen Perspektive David Eastons (1965) ist die Legitimation politischer Regime von entscheidender Bedeutung, um

PD Dr. S. Kailitz

Hannah-Arendt-Institut für Totalitarismusforschung, Helmholtzstraße 6, 01069 Dresden, Deutschland E-Mail: steffen.kailitz@mailbox.tu-dresden.de

Prof. Dr. S. Wurster $(\bowtie)$

Professur für Policy Analysis, Hochschule für Politik München, Technische Universität München, Richard-Wagner-Straße 1, 80333 München, Deutschland

E-Mail: stefan.wurster@hfp.tum.de 
ihre Funktionsweise und Dauerhaftigkeit zu verstehen (Alagappa 1995). Von einer besonderen Affinität der deutschen Politikwissenschaft zum Themenkomplex zeugt nicht zuletzt, dass die Deutsche Vereinigung für Politikwissenschaft ${ }^{1}$ sich in gleich zwei Kongressen mit den „Legitimationsproblemen politischer Ordnung“ (1976) ${ }^{2}$ und den „Legitimationsproblemen der Ordnung von Freiheit“ $(2015)^{3}$ beschäftigte.

Die Legitimation eines politischen Regimes zielt darauf, eine dauerhafte Basis für die Ausübung staatlicher Autorität zu gewährleisten. Zur Legitimation eines Regimes zählen dabei alle Maßnahmen eines politischen Regimes, die darauf zielen, freiwillige Gefolgschaft oder zumindest Fügsamkeit mithilfe politischer Verheißungen, Versprechungen und konkreter Maßnahmen zu erzeugen, unabhängig davon, ob diese erfolgreich sind, also ob es mittels dieser Verheißungen, Versprechungen und Maßnahmen gelingt, die Herrschaft tatsächlich durch die Erzeugung von Gefolgschaft zu stabilisieren. Mit Blick auf den in den Sozialwissenschaften mehrdeutig verwendeten Begriff der Legitimität eines politischen Regimes folgen die Beiträge in diesem Themenheft einer empirischen Interpretation des Legitimitätsbegriffs im Sinne des Legitimitätsglaubens von Max Weber. Die Legitimität eines Regimes bezieht sich in dieser Hinsicht auf die prinzipielle Anerkennung der Rechtmäßigkeit des politischen Regimes durch die Bürger und ihre damit verbundene Unterstützung (Kielmansegg 1971, S. 368) des Regimes, indem dessen Regeln, etwa Steuergesetze, befolgt werden. Wir verwenden für dieses Verständnis den Begriff der Legitimation. Andere haben im Anschluss an Easton den Begriff der Legitimität gewählt, obwohl er im Deutschen starke normative Konnotationen hat. Easton differenziert zwischen spezifischer Unterstützung (etwa deckungsgleich mit Output-Legitimität) ${ }^{4}$ und diffuser Unterstützung aufgrund eines Glaubens an die Legitimität des politischen Regimes (Easton 1965, S. 247-470). Ganz ähnlich - wenn auch (zu) stark konzentriert auf die demokratische Herrschaftsform - unterscheidet Seymour Martin Lipset im modernisierungstheoretischen Modell zwischen „Legitimität““ (= Input-Legitimität) und „Effektivität“ (= Output-Legitimität) als zentralen Komponenten um die Dauerhaftigkeit politischer Herrschaft $(1959,1960,1963,1984)$ zu erklären. Mit Blick auf die Quellen der Legitimität unterscheidet Easton wiederum zwischen ideologischen, strukturellen und personalen (Easton 1965, S. 286f.). Dabei kann das konkrete Mischungsverhältnis dieser Komponenten in konkreten politischen Regimen sehr unterschiedlich sein. Diese Ausführungen machen deutlich, dass auch Easton den Begriff der Legitimität im Sinne des Weberschen Legitimitätsglauben - also als Legitimation - versteht. Da Legitimität jedoch stets auch normative Geltungsansprüche stellt und somit die Akzeptanz von Herrschaft an bestimmte normativ rechtfertigbare Werte bindet, greifen wir diesen Begriff nicht auf.

\footnotetext{
1 Es handelt sich nicht um einen Schreibfehler. Die Vereinigung hat sich 2016 umbenannt.

2 Vgl. Kielmansegg (1976).

3 Vgl. Abels (2016).

4 In der neueren Literatur setzt sich der zutreffende Begriff „Output“-Legitimität erfreulicherweise durch (Schmidt 2012; Lindgren und Persson 2010). Mit dem häufig in der Vergangenheit synonym zu OutputLegitimität verwendeten Begriff Output-Legitimation wurde nämlich die Bezugsebene verwechselt, wenn der Begriff auf die Einstellungen von Bürgern zielte.
} 
Die Legitimation der Macht konstituiert und definiert in gewisser Weise letztlich ein politisches Regime (Barker 2001, S. 30; siehe auch Beetham 1991). Die Bedeutung der „Legitimitätspolitik“ (Geis et al. 2012) politischer Regime hat im Zeitverlauf mit dem wachsenden Einfluss von Bürgern auf die Politik noch zugenommen, weil Bürger inzwischen selbst in Autokratien gewöhnlich nicht mehr lediglich Herrschaftsunterworfene sind. Die Legitimation der Herrschaft richtet sich aber keineswegs nur an die ,einfachen“ Bürger, um Legitimitätsgauben oder zumindest eine Hinnahme der Herrschaft zu erwirken, sondern sie dient auch der Selbstrechtfertigung der Herrschaftselite und vor allem des Autokraten selbst (Barker 2001). Tatsächlich gibt es in der Legitimationsforschung seit Max Weber sogar eine Neigung die vorrangige Bedeutung der Regimelegitimation nicht in einer Überzeugung der ,einfachen“ Bürger von der Legitimität des Regimes zu sehen, sondern darin, dass sie eine Bindewirkung für die entscheidenden Angehörigen des Herrschaftsapparats erzeugt und mithin die Regimestabilität fördert (so etwa Beetham und Lord 1998, S. 10). Die Legitimationsforschung hilft zu verstehen, wie politische Regime entstehen, funktionieren und untergehen. Prinzipiell lässt sich zwischen der Rechtfertigung eines Regimewechsels und der Legitimationsbasis eines darauf folgenden neuen Regimes unterscheiden (Beetham 2013, S. 232). In Regimekrisen trifft der Legitimitätsanspruch der Herrschenden auf dessen fundamentale Zurückweisung durch die Regimeopposition. So gilt etwa eine Legitimationskrise der kommunistischen Regime (Di Palma 1991; Holmes 1993, 1997) als ein zentraler Erklärungsfaktor für den Untergang der kommunistischen Regime 1989 bis 1991. Eine Regimekrise kann mit einem Überleben des alten Regimes und damit verbunden einer Neujustierung der Legitimationsbasis des Regimes enden oder mit einer Systemtransformation zu einem neuen politischen Regime, dass seine Legitimationsbasis erst etablieren muss.

\section{Legitimation in Autokratien}

Von einer normativ-liberaldemokratischen Perspektive betrachtet, gelten Autokratien noch immer häufig pauschal als gänzlich illegitim (Dogan 1992) und damit ist die Diskussion über die mögliche Legitimation autokratischer Regime bereits beendet. Auf der empirischen Ebene ist aber beobachtbar, dass auch Autokratien über ein bedeutendes Maß an empirischer Gefolgschaft (ob wir dies nun Legitimität oder anders nennen) und vor allem einem tatsächlichen Legitimitätsglauben unter den Angehörigen des Herrschaftsapparats verfügen können. Einige gegenwärtige autokratische Regime wie China oder Russland unter Putin erscheinen Länderexperten geradezu als ,ppopulär“ (Brady 2009; Ross 2014). Zu Unrecht wird dabei das Phänomen des ,populären Autoritarismus“ noch immer als paradox angesehen. Der Blick auf die enorme Popularität der Diktatoren Juan Perón (Schoultz 1977) in Argentinien in den 1970er-Jahren und Adolf Hitler in Deutschland (Kershaw 2009) in den 1930er-Jahren zeigt, dass das Phänomen des ,,populären“ Autokraten keineswegs neu ist. Während manche Autokratien darauf setzen, einen „Mythos“ um den ,charismatischen“ „Führer“ (Kershaw 1980) oder ,lider“ (Hoffmann 2009) zu kreieren, setzen andere Autokratien aber stärker auf institutionell ausgerichtete Legitimationsstrategien. Die offiziellen Legitimationsstrategien sind in der Regel dabei 
auch in Autokratien keineswegs bloße „Schaufensterdekorationen“. Die Regimeelite selbst orientiert sich zumindest in ihrem Handeln auf der politischen Bühne an diesen Legitimationsstrategien (Barker 2001). Geschieht dies nicht, drohen Legitimationskrisen (Beetham 1991).

Wie jedes politische Regime bedürfen auch Autokratien, um dauerhaft zu sein, also einer belastbaren Legitimationsstrategie. Allein auf Repression zu setzen ist für den Autokraten nämlich teuer und zudem auch potentiell gefährlich. Je mehr der Autokrat einseitig auf den Unterdrückungsapparat setzt, desto gefährdeter ist er durch genau diesen Unterdrückungsapparat gestürzt zu werden (u. a. Svolik 2012, S. 10). Kooptation, verstanden als selektive Einbindung oppositioneller Teile der Eliten durch persönliche Begünstigungen, birgt wiederum die Gefahr, dass die begünstigten Personen genau diese Ressourcen nutzen, um den Autokraten zu stürzen. Daher ist die (zusätzliche) Legitimation autokratischer Herrschaft von großer Bedeutung.

Legitimation in Autokratien kann sich auf verschiedene Ebene beziehen. Die Legitimation kann 1. auf die übergeordnete Ebene des Nationalstaats, 2. direkt auf das autokratische Regime selbst, 3. auf konkrete Herrschaftsträger und/oder 4. auf konkrete politische Entscheidungen zielen. Dass eine bestimmte politische Entscheidung als illegitim angesehen wird, muss dabei noch nicht bedeuten, dass die Herrschaftsträger als illegitim gelten. Gelten konkrete Herrschaftsträger etwa ein bestimmter Thronfolger in einer Monarchie (z. B. wegen Geisteskrankheit oder zweifelhafter Herkunft) als illegitim, bedeutet dies keineswegs zwangsläufig, dass die Regimeform der Monarchie als illegitim angesehen wird. Gilt aber die Regimeform selbst als illegitim, ist es schwierig, mehr als eine bloße Hinnahme der Herrschaftsträger und ihrer politischen Entscheidungen zu erreichen.

Die Beiträge in diesem Themenheft konzentrieren sich schwerpunktmäßig auf die Versuche der Legitimation autokratischer Regimeformen, also nicht bloß der Legitimation konkreter Herrschaftsträger oder konkreter politischer Entscheidungen. Allerdings sind die Grenzen oft fließend und spätestens beim Blick auf personalistische Regime ist die Grenze zwischen einer Legitimation der Person des Autokraten und des autokratischen Regimes nicht mehr trennscharf zu ziehen.

Autokratien haben nun verschiedene Möglichkeiten der Regimelegitimation. Diese reichen von der vergangenheitsorientierten monarchistisch-traditionalen Begründung (Herb 1999) über gegenwartsorientierte Legitimation über die Verfahren und Institutionen durch die Entscheidungen getroffen und kontrolliert werden (Luhmann 1969; Habermas 1973) bis hin zu zukunftsorientiert ideologisch-utopischen Versuchen Herrschaft zu rechtfertigen (Backes und Kailitz 2014c; Rigby und Fehér 1982; Pakulski 1987). Es gibt grundlegend verschiedene Legitimationsstrategien je nach Regimevariante der Autokratie (Kailitz 2013a; Mayer 2001). Diese Legitimationsstrategien können relativ vage wie etwa im Falle vieler Militärautokratien ausfallen oder sehr ausgearbeitet sein wie etwa im Falle der kommunistischen Ideokratien (Backes und Kailitz 2016). Nicht jeder Anspruch auf ein Recht zur Herrschaft führt auch gleichermaßen zu einem Legitimitätsglauben der Bevölkerung (Gilley 2006, 2009; Hudson 1977) und/oder der Angehörigen des Herrschaftsapparats. Insbesondere da nicht jede Legitimationsstrategie in gleicher Stärke zu einem Zusammenhalt 
der autokratischen Elite beiträgt, wirkt sich die gewählte Legitimationsstrategie auf die Dauerhaftigkeit autokratischer Herrschaft aus.

Gerade die - allerdings nur verbal - eng an der Legitimationsstrategie liberaler Demokratien orientierte Legitimationsstrategie elektoraler Autokratien ist für Autokraten ein zweischneidiges Schwert. Sie erscheint als ihre letzte Verteidigungslinie (Schedler 2010). Autokraten sehen sich demnach um die Reputation im Innern und Äußern zu erhöhen zu einer verbal-demokratischen Legitimation gezwungen. Damit riskieren sie aber an einem Maßstab gemessen zu werden, den sie gar nicht erfüllen können. Der Traum von Autokraten ist es, die Früchte demokratischer Legitimität genießen zu können, ohne die Gefahr ihrer Abwahl einzugehen (Schedler 2002, S. 37). Sie wollen damit letztlich den Kreis quadrieren. Die demokratische Legitimation erfordert nämlich eine stetige prozedurale Legitimation des Regimes durch eine Fairness der Verfahren und Institutionen. Wenn sich nun eine Kluft auftut, zwischen den propagierten und tatsächlichen Verfahren, dann liefern Wahlen wie in elektoralen Autokratien keine verlässliche prozedurale Legitimation. Es ist daher fraglich, ob sie unter diesen Rahmenbedingungen tatsächlich wirksam zur Dauerhaftigkeit des politischen Regimes beitragen (Beetham 1991). Allerdings ist nicht außer Acht zu lassen, dass Wahlen neben der inneren und äußeren Legitimation von Autokratien auch noch weitere Funktionen erfüllen können, etwa eine Informationsund Signalfunktion, bei der die autokratische Eliten mittels Wahlen Präferenzen der Bevölkerung erkundet.

Autokratien können verschiedene Instrumente auf der Input- und Output-Seite zu ihrer Legitimation und zum Gewinn von Unterstützung durch die Bevölkerung einsetzen, wie ein manipuliertes Rechtssystem (der Beitrag von Lauth in diesem Heft; Myint 2014; Rajah 2012) oder Massenmedien (King et al. 2013). Institutionen und geregelte Verfahren, die in der Herrschaftspraxis auch tatsächlich Bedeutung haben, können in Autokratien wesentlich zur Legitimation politischer Herrschaft beitragen.

Die gegenwärtige Debatte ist, wenn auf die Legitimationskraft von Institutionen und Verfahren eingegangen wird, aber noch zu stark auf die Legitimationswirkung „demokratischer“ Institutionen und Verfahren in Autokratien fixiert. Unzureichend erforscht sind aber etwa die Legitimationswirkung von spezifisch nicht-demokratischen Institutionen wie etwa dem Adel in Monarchien (zu den Ausnamen zählt Roller 2001). Auch die Wirkung von ,traditionellen“ außerpolitischen Legitimationsquellen wie Religion (Smith 1978), traditionellen Werte (Schatzberg 2001), Nationalismus (Breslin 2008) und ethnische Identität sowie die Bedeutung des Charisma oder der vergangenen politischen Leistungen eines Herrschers (Eatwell 2006; Hoffmann 2009) - etwa bei der Nationalstaatsbildung sind stärker als bislang bei der Betrachtung der Legitimationsstrategien von Autokratien zu beachten. Unzureichend in den Blick genommen ist bislang in Bezug auf Autokratien auch ein zentraler Topoi der Legitimitätsforschung, nämlich inwiefern rational-legale Verfahren und überhaupt Legalität Legitimation (Luhmann 1969; Habermas 1973) erzeugen kann. Welche Bedeutung haben namentlich - formal - rechtsstaatliche Strukturen im Sinne einer rule by law (Ginsburg und Moustafa 2008; Wang 2016; Myint 2014; Pereira 2005) in Autokratien? 
Neben der ideologischen Legitimation und der Legitimation durch politische Teilhabe der Bürger kann sich ein politisches Regime schließlich auch über eine sehr gute Policy-Performance (Output-Legitimation) legitimieren (Easton 1965; Scharpf 2004; Lipset 1959). Bislang wurde hauptsächlich untersucht, inwiefern bestimmte Autokratietypen eine bessere Policy-Performance erbringen als andere Autokratietypen und die liberale Demokratie (Croissant et al. 2014; Kailitz 2013b; Knutsen 2011; McGuire 2013; Schmidt 2013; Stockemer 2013; Wu 2012; Wurster 2013). Relativ selten wurden dabei bislang direkt Überlegungen zu den Auswirkungen der Regimelegitimation auf die Policy-Leistungen und umgekehrt angestellt (zu den Ausnahmen zählen Buzogány et al. 2016; Kailitz 2013b; Schmidt 2012; Kailitz und Wurster 2016). Der Volksrepublik China oder Singapur - aber etwa auch weniger bekannten Fällen wie Vietnam - scheint es recht gut zu gelingen, sich über ein hohes Wirtschaftswachstum und einen steigenden Wohlstand zu legitimieren (Heberer und Schubert 2008; Hiep 2012). Kommunistische Regime in der Zeit des „Kalten Krieges“ wie Kuba oder die Sowjetunion versuchten dagegen stärker über eine breite soziale Absicherung, etwa mittels eines Rechts auf Arbeit, Unterstützung zu erlangen (u. a. Cook 1993; Schmidt 2004). Stärkere Aufmerksamkeit verdienen auch die Versuche von Autokratien, sich über außenpolitische Erfolge zu legitimieren (Holbig 2011; Kneuer 2013). Im Unterschied zur Legitimation über die „Heiligkeit“ der Herkunft der Herrschaftsträger oder ihrer ideologischen Ziele (Griffin 2012) sowie der Legitimation durch rational-legale Verfahren und Institutionen ist es für Autokraten allerdings potentiell gefährlich, sich bloß auf Output-Legitimation zu stützen. Sie machen sich damit abhängig von einer zumindest gleichbleibend guten PolicyPerformance (Holbig 2001).

\section{Fragestellung und innovatives Potential des Themenheftes}

Dieses Themenheft der Zeitschrift für vergleichende Politikwissenschaft adressiert erstmals die Legitimation in Autokratien in einer systematischen Weise von sehr verschiedenen Blickwinkeln in einer in sich geschlossenen Publikation. ${ }^{5}$ Dies verspricht einen bedeutenden Erkenntnisgewinn mit Blick auf die Funktionsweise von Autokratien. Wir wollen dazu beitragen, Antworten auf vier eng miteinander verknüpfte Leitfragen zu finden:

1. In welchem Verhältnis steht die Legitimation zu Repression und Kooptation, den beiden anderen zentralen Instrumenten von Autokraten, um ihre Herrschaft zu stabilisieren? Ist das Verhältnis von Legitimation, Kooptation und Repression in allen Autokratien gleich oder gibt es Unterschiede je nach Autokratietyp? Mit dieser Fragestellung führen wir eine sich bereits lebhaft in Gang befindliche Debatte außerhalb (Backes und Kailitz 2014a, 2014b; Gerschewski 2013, 2014a, 2014b) und innerhalb der ZfVP fort (Lueders und Croissant 2014, 2015; Kailitz und Tanneberg 2015).

\footnotetext{
5 Begrenzt auf den postsowjetischen Raum wurde inzwischen immerhin eine sehr anregender Sammelband zu der Thematik vorgelegt: Brusis (2016).
} 
2. Welche konkreten Legitimationsstrategien verfolgen Autokratien und zu welchem Zweck geschieht dies?

3. Wie wirken sich die Legitimationsstrategien von Autokraten auf den Legitimitätsglauben und das Verhalten der ,normalen“ Bürger, der Regimeelite und der Opposition sowie die Stabilität des politischen Regimes im Ganzen aus?

4. Wie sind die ideelle Regimelegitimation, die Legitimation durch Institutionen und Verfahren (Input-Legitimation im weitesten Sinne) und die Outputlegitimation durch Policy-Leistungen in Autokratien miteinander verwoben?

Die Leitfragen werden in acht Beiträgen aus unterschiedlichen Blickwinkeln behandelt. In einem abschließenden Fazit führen die Herausgeber die Fäden der Beiträge zusammen und erörtern, welchen Beitrag das Themenheft zu Antworten auf diese Fragen leistet. Zudem werden knapp Perspektiven für die Forschung zur Legitimation und Legitimität in Autokratien skizziert. Beim Thema Autokratien handelt es sich um ein für alle Unterdisziplinen der Politikwissenschaft relevantes Thema, das nicht nur für die in diesem Heft gewählte komparative Perspektive bedeutsam ist. Für die Vergleichende Politikwissenschaft handelt es sich wie bereits eingangs erörtert um ein Kernthema und sollte für alle die in diesem Bereich arbeiten von Interesse sein. Für Vertreter der Internationalen Politik ist das Heft vor allem mit Blick auf die Bedeutung des Verhaltens externer Akteure für die Legitimation von Autokratien und die Bedeutung der Außenpolitik für die Legitimationsstrategien in Autokratien interessant. Für Vertreter der Politischen Ökonomie erscheint vor allem der Blick auf die Verbindungslinie zwischen der Regimelegitimation und sozial- und wirtschaftspolitischen Policy-Leistungen bedeutsam. Für politische Theoretiker ist vor allem der Teil zu den konzeptionellen und theoretischen Grundlagen der Legitimation in Autokratieforschung relevant. Neben dem Interesse aus allen Bereichen der Politikwissenschaft erhoffen wir ein bedeutendes Interesse aus den Nachbardisziplinen der Soziologie und der Volkswirtschaftslehre (Teilbereich politische Ökonomie).

Zum Gelingen dieses Themenheftes haben neben den Autorinnen und Autoren auch die Diskutanten der Tagung „Legitimationsstrategien von Autokratien“ vom 22. und 23. Oktober 2015 in Dresden maßgeblich beigetragen. Als Ausrichter der Tagung fungierte das Hannah-Arendt-Institut für Totalitarismusforschung (HAIT) an der TU Dresden. Weiterhin bedanken sich die Herausgeber sehr herzlich bei der Fritz Thyssen-Stiftung für die großzügige Finanzierung der Tagung.

\section{Literatur}

Abels, Gabriele. 2016. Vorsicht Sicherheit! Legitimationsprobleme der Ordnung von Freiheit. 26. wissenschaftlicher Kongress der Deutschen Vereinigung für Politische Wissenschaft. Baden-Baden: Nomos.

Abulof, Uriel. 2015. 'Can't buy me legitimacy': the elusive stability of Mideast rentier regimes. Journal of International Relations and Development doi:10.1057/jird.2014.32.

Alagappa, Muthiah. 1995. Seeking a more durable basis of authority. In Political Legitimacy in Southeast Asia: The Quest for Moral Authority, Hrsg. Muthiah Alagappa, 293-334. Stanford: Stanford University Press.

Backes, Uwe. 2013. Vier Grundtypen der Autokratie und ihre Legitimierungsstrategien. In Autokratien im Vergleich. Sonderheft der Politische Vierteljahresschrift 47/2012, Hrsg. Steffen Kailitz, Patrick Köllner, 157-176. Baden-Baden: Nomos. 
Backes, Uwe, und Steffen Kailitz. 2014a. Einleitung. In Ideokratien im Vergleich. Legitimation, Kooptation und Repressio, Hrsg. Uwe Backes, Steffen Kailitz, 7-16. Göttingen: Vandenhoek \&amp; Ruprecht.

Backes, Uwe, und Steffen Kailitz. 2014b. Ergebnisse. In Uwe Backes, und Steffen Kailitz, Hrsg. Ideokratien im Vergleich. Legitimation, Kooptation, und Repression, 383-402. Göttingen: Vandenhoek \& Rupr echt.

Backes, Uwe, und Steffen Kailitz. 2014c. Ideokratien im Vergleich. Legitimation, Kooptation und Repression. Göttingen: Vandenhoek \&amp; Ruprecht.

Backes, Uwe, und Steffen Kailitz. 2016. Ideocracies in comparison. Legitimation, cooptation, and repression. London: Routledge.

Barker, Rodney. 2001. Legitimating identities: The self-presentation of rulers and subjects. Cambridge: Cambridge University Press.

Beetham, David. 1991. The legitimation of power. Basingstoke: Macmillan.

Beetham, David. 2013. The legitimation of power, 2. Aufl., Basingstoke: Macmillan.

Beetham, David, und Christopher Lord. 1998. Legitimacy and the EU. Essex [England]: Longman.

Brady, Anne-Marie. 2009. Mass persuasion as a means of legitimation and China's popular authoritarianism. American Behavioral Scientist 53(3):434-457. doi:10.1177/0002764209338802.

Breslin, Shaun. 2008. Democratising one-party rule? Political reform, nationalism and legitimacy in the People's Republic of China. Madrid: FRIDE.

Brusis, Martin. 2016. The politics of legitimation in post-soviet Eurasia. In Politics and Legitimacy in PostSoviet Eurasia, Hrsg. Martin Brusis, Joachim Ahrens, und Martin Schulze Wessel, 1-17. London: Palgrave.

Burnell, Peter. 2006. Autocratic opening to democracy: Why legitimacy matters. Third World Quarterly 27(4):545-562.

Buzogány, Aron, Rolf Frankenberger, und Patricia Graf. 2016. Policy-Making und Legitimation in Autokratien: Das Beispiel der Innovationspolitik. Totalitarismus und Demokratie 13(2):257-280.

Connolly, William E. 1984. Legitimacy and the State. Oxford: Blackwell.

Cook, Linda J. 1993. The Soviet social contract and why it failed: Welfare policy and workers' politics from Brezhnev to Yeltsin. Cambridge: Harvard University Press.

Croissant, Aurel, Steffen Kailitz, Patrick Köllner, und Stefan Wurster. 2014. Comparing autocracies in the early twenty-first century. The performance and persistence of autocracies, Bd. 2. London: Routledge.

Denitsch, Bogdan. 1979. Legitimation of regimes. International frameworks for analysis. London: Sage.

Di Palma, Giuseppe. 1991. Legitimation from the top to civil society: Politico-cultural change in eastern Europe. World Politics 44(1):49-80.

Dogan, Mattei. 1992. Conceptions of legitimacy. In Encyclopedia of government and politics, Hrsg. M.E. Hawkesworth, Maurice Kogan, 116-126. London: Routledge.

Dukalskis, Alexander. 2017. The authoritarian public sphere: Legitimation and autocratic power in North Korea, Burma, and China. London: Routledge.

Easton, David. 1965. A systems analysis of political life. New York: Wiley.

Eatwell, Roger. 2006. The concept and theory of charismatic leadership. Totalitarian Movements \& Political Religions 7(2):141-156.

Gandhi, Jennifer. 2008. Political institutions under dictatorship. Cambridge: Cambridge University Press.

Geddes, Barbara. 1999. What do we know about democratization after twenty years? Annual Review of Political Science 2:115-144.

Geis, Anna, Frank Nullmeier, und Christopher Daase. 2012. Der Aufstieg der Legitimitätspolitik. Rechtfertigung und Kritik politisch-ökonomischer Ordnungen. Baden-Baden: Nomos.

Gerschewski, Johannes. 2013. The three pillars of stability: Legitimation, repression, and co-optation in autocratic regimes. Democratization 20(1):13-38. doi:10.1080/13510347.2013.738860.

Gerschewski, Johannes. 2014a. Legitimation, Kooptation und Repression in Ideokratein aus der Perspektive des historischen Institutionalismus. In Ideokratien im Vergleich. Wechselbeziehungen zwischen Legitimation, Kooptation und Repression, Hrsg. Uwe Backes, Steffen Kailitz, 95-112. Göttingen: Vandenhoeck \& Ruprecht.

Gerschewski, Johannes. 2014b. Stabilizing autocratic rule. Legitimation, repression, and co-optation in East Asia's autocracies. Berlin: Humboldt-Universität zu Berlin.

Gilley, Bruce. 2006. The meaning and measure of state legitimacy: Results for 72 countries. European Journal of Political Research 45(3):499-525.

Gilley, Bruce. 2009. The right to rule: How states win and lose legitimacy. New York: Columbia University Press.

Ginsburg, Tom, und Moustafa Tamir. 2008. Rule by law: The politics of courts in authoritarian regimes. Cambridge: Cambridge University Press. 
Griffin, Roger. 2012. The legitimizing role of palingenetic myth in ideocracies. Totalitarismus und Demokratie 9(1):39-56.

Habermas, Jürgen. 1973. Legitimationsprobleme im Spätkapitalismus. Frankfurt a.M.: Suhrkamp.

Hanson, Stephen E. 2011. Plebiscitarian patrimonialism in Putin's Russia: Legitimating authoritarianism in a postideological era. The Annals of the American Academy of Political and Social Science 636(1):32-48. doi:10.1177/0002716211398210.

Heberer, Thomas, und Gunter Schubert. 2008. Regime legitimacy in contemporary China: Institutional change and stability. London: Routledge.

Herb, Michael. 1999. All in the family, absolutism, revolution and democracy in the middle eastern monarchies. New York: State University of New York Press.

Hiep, Le Hong. 2012. Performance-based legitimacy: The case of the communist party of Vietnam and Doi Moi. Contemporary Southeast Asia 34(2):145-172.

Hoffmann, Bert. 2009. Charismatic authority and leadership change: Lessons from Cuba's post-Fidel succession. International Political Science Review 30(3):229-248. doi:10.1177/0192512109105635.

Holbig, Heike. 2001. Inflation als Herausforderung der Legitimation politischer Herrschaft in der VR China: Wirtschaftspolitische Strategien in den Jahren 1987-1989. Hamburg: Institut für Asienkunde.

Holbig, Heike. 2013. Ideology after the end of ideology. China and the quest for autocratic legitimation. Democratization 20(1):61-81. doi:10.1080/13510347.2013.738862.

Holbig, Heike. 2011. International dimensions of legitimacy: Reflections on western theories and the chinese experience. Journal of Chinese Political Science 16:161-181.

Holmes, Leslie. 1993. The end of communist power: Anti-corruption campaigns and legitimation crisis. Oxford: Oxford University Press.

Holmes, Leslie. 1997. Post-communism: An introduction. Durham: Duke University Press.

Hudson, Michael C. 1977. Arab politics: The search for legitimacy. New Haven: Yale University Press.

Kailitz, Steffen. 2013a. Classifying political regimes revisited: Legitimation and durability. Democratization 20(1):38-59.

Kailitz, Steffen. 2013b. Macht der Autokratietyp einen Unterschied für das Wirtschaftswachstum? In $A u$ tokratien im Vergleich. Sonderheft der Politische Vierteljahresschrift 47/2012, Hrsg. Steffen Kailitz, Patrick Köllner, 500-527. Baden-Baden: Nomos.

Kailitz, Steffen, und Patrick Köllner. 2013. Zur Autokratieforschung der Gegenwart: Klassifikatorische Vorschläge, theoretische Ansätze und analytische Dimensionen. In Autokratien im Vergleich. Sonderheft der Politischen Vierteljahresschrift 2012, Hrsg. Steffen Kailitz, Patrick Köllner, 9-34. BadenBaden: Nomos.

Kailitz, Steffen, und Daniel Stockemer. 2015. Regime legitimation, elite cohesion and the durability of autocratic regime types. International Political Science Review doi:10.1177/0192512115616830.

Kailitz, Steffen, und Dag Tanneberg. 2015. Legitimation, Kooptation, Repression und das Überleben von Autokratien ,im Umfeld autokratischer Wahlen“. Eine Replik auf den Beitrag von Hans Lueders und Aurel Croissant. Zeitschrift für Vergleichende Politikwissenschaft. Comparative Governance and Politics 9(1-2):73-82. doi:10.1007/s12286-015-0233-1.

Kailitz, Steffen, und Stefan Wurster. 2016. Regimelegitimation und Gesundheitspolitik in Autokratien. In Vorsicht Sicherheit! Legitimationsprobleme der Ordnung von Freiheit: 26. wissenschaftlicher Kongress der Deutschen Vereinigung für Politische Wissenschaft, Hrsg. Gabriele Abels, 131-154. BadenBaden: Nomos.

Kershaw, Ian. 1980. Der Hitler-Mythos: Volksmeinung und Propaganda im Dritten Reich. Stuttgart: Deutsche Verlags-Anstalt.

Kershaw, Ian. 2009. Consensus, coercion and popular opinion in the Third Reich: Some reflections. In Popular opinion in totalitarian regimes: Fascism, nazism, communism, Hrsg. Paul Corner, 32-46. Oxford: Oxford University Press.

Graf Kielmansegg, Peter. 1971. Legitimität als analytische Kategorie. Politische Vierteljahresschrift 12:360-401.

Graf Kielmansegg, Peter. 1976. Legitimationsprobleme politischer Systeme. Sonderheft 7 der Politischen Vierteljahresschrift. Opladen: Westdeutscher Verlag.

King, Gary, Jennifer Pan, und Margaret E. Roberts. 2013. How censorship in China allows government criticism but silences collective expression. American Political Science Review 107(02):326-343. doi:10.1017/S0003055413000014.

Kneuer, Marianne. 2013. Die Suche nach Legitimität. Außenpolitik als Legitimationsstrategie autoritärer Regime. In Autokratien im Vergleich. Sonderheft der Politische Vierteljahresschrift 47/2012, Hrsg. Steffen Kailitz, Patrick Köllner, 205-236. Baden-Baden: Nomos.

Knutsen, Carl Henrik. 2011. The economic effects of democracy and dictatorship. Oslo: University of Oslo. 
Köllner, Patrick, und Steffen Kailitz. 2013. Comparing autocracies: Theoretical issues and empirical analyses. Democratization 20(1):1-12. doi:10.1080/13510347.2013.738859.

Lindgren, Karl-Oskar, und Thomas Persson. 2010. Input and output legitimacy: Synergy or trade-off? Empirical evidence from an EU survey. Journal of European Public Policy 17(4):449-467.

Lipset, Seymour Martin. 1959. Some social requisites of democracy: Economic development and political legitimacy. American Political Science Review 53:69-105.

Lipset, Seymour Martin. 1960. Political man: The social basis of politics. Garden City: Anchor Books.

Lipset, Seymour Martin. 1963. The first new Nation. The United States in historical and comparative perspective. New York: Basic Books.

Lipset, Seymour Martin. 1984. Social conflict, legitimacy, and democracy. In Legitimacy and the state, Hrsg. William E. Connolly, 88-103. Oxford: Blackwell. Readings in social and political theory.

Lueders, Hans, und Aurel Croissant. 2014. Wahlen, Strategien autokratischer Herrschaftssicherung und das Überleben autokratischer Regierungen. Zeitschrift für Vergleichende Politikwissenschaft. Comparative Governance and Politics 8:329-355.

Lueders, Hans, und Aurel Croissant. 2015. Eine Antwort auf die Replik von Kailitz und Tanneberg zu unserem Beitrag „Wahlen, Strategien autokratischer Herrschaftssicherung und das Überleben autokratischer Regierungen“. Zeitschrift für Vergleichende Politikwissenschaft. Comparative Governance and Politics 9(3):183-193.

Luhmann, Niklas. 1969. Legitimation durch Verfahren. Frankfurt a.M.: Suhrkamp.

Mayer, Robert. 2001. Strategies of justification in authoritarian ideology. Journal of Political Ideologies 6(2):147-168.

McGuire, James W. 2013. Political regime and social performance. Contemporary Politics 19(1):55-75. doi:10.1080/13569775.2013.773203.

Morgenbesser, Lee. 2015. In search of stability. Electoral legitimation under authoritarianism in Myanmar. European Journal of East Asian Studies 14(2):163-188.

Myint, Aung P. 2014. Legal hybridity: Rule of law under authoritarianism. Lewiston: Bates College.

Pakulski, Jan. 1987. Ideology and political domination. A critical re-appraisal. International Journal of Comparative Sociology 28(3):129-157. doi:10.1163/156854287X00112.

Pepinsky, Thomas. 2013. The institutional turn in comparative authoritarianism. British Journal of Political Science. doi:10.1017/S0007123413000021.

Pereira, Anthony W. 2005. Political (in)justice. authoritarianism and the rule of law in Brazil, Chile, and Argentina. Pittsburgh: University of Pittsburgh Press.

Pickel, Gert. 2013. Die kulturelle Verankerung von Autokratien. Bestandserhalt durch ideologische Legitimationsstrategien und ökonomische Legitimität oder Demokratisierung? In Autokratien im Vergleich. Sonderheft der Politische Vierteljahresschrift 47/2012, Hrsg. Steffen Kailitz, Patrick Köllner, 205-236. Baden-Baden: Nomos.

Rajah, Jothie. 2012. Authoritarian rule of law: Legislation, discourse, and legitimacy in Singapore. Cambridge: Cambridge University Press.

Rigby, Thomas H., und Ferenc Fehér. 1982. Political legitimation in communist states. New York: St. Martin Press.

Roller, Matthew B. 2001. Constructing autocracy: Aristocrats and emperors in Julio-Claudian Rome. Princeton: Princeton University Press.

Ross, Cameron. 2014. Das Paradox: Putins populäre Autokratie. Legitimitätsquellen in einem hybriden Regime. Osteuropa 64(8):99-112.

Scharpf, Fritz W. 2004. Legitimationskonzepte jenseits des Nationalstaats. Köln: Max-Planck-Institut für Gesellschaftsforschung.

Schatzberg, Michael G. 2001. Political legitimacy in middle Africa: Father, family, food. Bloomington: Indiana University Press.

Schedler, Andreas. 2002. Elections without democracy. The menu of manipulation. Journal of Democracy 13(2):36-50.

Schedler, Andreas. 2009. The new institutionalism in the study of authoritarian regimes. Totalitarismus und Demokratie 6:327-344.

Schedler, Andreas. 2010. Authoritarianism's last line of defense. Journal of Democracy 21(2):69-80.

Schlumberger, Oliver. 2010. Opening old bottles in search of new wine: On nondemocratic legitimacy in the middle east. Middle East Critique 19(3):233-250.

Schmidt, Manfred G. 2004. Sozialpolitik der DDR. Sozialpolitik und Sozialstaat. Wiesbaden: VS Verlag für Sozialwissenschaften.

Schmidt, Manfred G. 2012. Legitimation durch Performanz? Zur Output-Legitimität in Autokratien. Totalitarismus und Demokratie 9(1):83-100. 
Schmidt, Manfred G. 2013. Staatstätigkeit in Autokratien und Demokratien. In Autokratien im Vergleich. Sonderheft der Politische Vierteljahresschrift 47/2012, Hrsg. Steffen Kailitz, Patrick Köllner, 418-438. Baden-Baden: Nomos.

Schoultz, Lars. 1977. The socio-economic determinants of popular-authoritarian electoral behavior: The case of Peronism. The American Political Science Review 71(4):1423-1446. doi:10.2307/1961488.

Smith, Bardwell L. 1978. Religion and legitimation of power in Thailand, Laos, and Burma. Chambersburg: ANIMA Books.

Stockemer, Daniel. 2013. Autokratien und Regierungsqualität: Eine vergleichende Analyse. In Autokratien im Vergleich. Sonderheft der Politische Vierteljahresschrift 47, Hrsg. Steffen Kailitz, Patrick Köllner, 477-499. Baden-Baden: Nomos.

Svolik, Milan W. 2012. The politics of authoritarian rule. Cambridge: Cambridge University Press.

Wang, Yuhua. 2016. Tying the autocrat's hands. The rise of the rule of law in China. Cambridge: Cambridge University Press.

Weber, Max. 1980. Wirtschaft und Gesellschaft. Grundriß der verstehenden Soziologie, 5. Aufl., Tübingen: Mohr.

Weber, Max. 1984. Legitimacy, politics, and the state. In Legitimacy and the state, Hrsg. William E. Connolly, 63-87. Oxford: Blackwell. Readings in social and political theory.

Wu, Chin. 2012. When is democracy better for economic performance and when is it not: The interaction between polity and structural factors. Studies in Comparative International Development 47(4):365-388. doi:10.1007/s12116-012-9118-3.

Wurster, Stefan. 2013. Comparing ecological sustainability in autocracies and democracies. Contemporary Politics 19(1):76-93. doi:10.1080/13569775.2013.773204. 